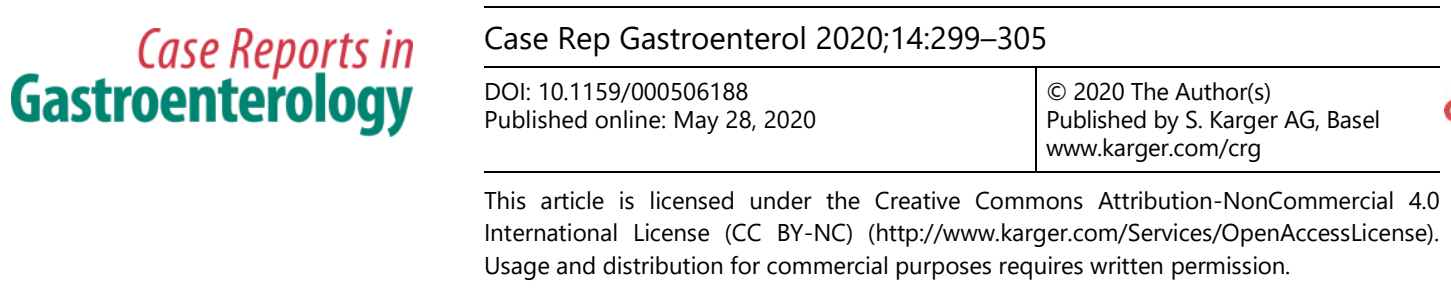

\title{
Synchronous Hepatocellular Carcinoma in a Patient with Primary Gastric Cancer: An Exceptional Association
}

\author{
Aya Hammami ${ }^{a} \quad$ Nour Elleuch $^{\mathrm{a}}$ Hanen Jaziri $^{\mathrm{a}}$ Yasser Ben Cheikh ${ }^{\mathrm{b}}$ \\ Ahlem Braham ${ }^{a}$ Salem Ajmi ${ }^{a} \quad$ Aida Ben Slama $^{a} \quad$ Mehdi Ksiaa $^{a}$ \\ Ali Jmaa ${ }^{a}$ \\ aDepartment of Gastroenterology, Faculty of Medicine, Ibn Al Jazzar, University Hospital \\ of Sahloul, Sousse, Tunisia; ${ }^{b}$ Department of Medical imaging, Faculty of Medicine, Ibn Al \\ Jazzar, University Hospital of Sahloul, Sousse, Tunisia
}

\section{Keywords}

Hepatocellular carcinoma - Gastric adenocarcinoma - Transarterial chemoembolization

\begin{abstract}
Hepatocellular carcinoma (HCC) is the most frequent type of liver cancer. Liver cirrhosis of any etiology is considered the main risk factor for the development of HCC. However, HCC in noncirrhotic livers remains an uncommon finding. The association of $\mathrm{HCC}$ with a primary gastric adenocarcinoma was described in the literature as part of a hepatoid adenocarcinoma which is a special type of primary gastric carcinoma characterized by histologic similarities to $\mathrm{HCC}$ with excessive production of $\alpha$-fetoprotein. Herein, we report the case of a 50 -year-old male patient, with no history of pre-existing liver disease, who was admitted due to epigastric pain and vomiting. He was diagnosed with HCC in noncirrhotic liver associated with primary gastric adenocarcinoma. To our knowledge, this is the first case report of synchronous HCC and gastric cancer with no hepatoid adenocarcinoma features in Tunisia.
\end{abstract}

(C) 2020 The Author(s)

Published by S. Karger AG, Basel 


\section{Introduction}

Worldwide, gastric cancer is the fourth most commonly diagnosed cancer and the second most common cause of cancer-related death [1,2]. Its incidence is twice as high in men as in women and increases with age. The median age of diagnosis is 70 years for men and 74 years for women [3].

Hepatocellular carcinoma (HCC) is the second leading cause of cancer-related death worldwide and the most frequent type of liver malignancy [4]. Liver cirrhosis of any etiology is considered as the major risk factor. However, HCC in noncirrhotic livers remains a rare condition. Besides the fibrolamellar variant, HCC on a healthy liver remains exceptional [5]. Synchronous gastric carcinoma and HCC is a rare entity that was described, in few cases, as hepatoid adenocarcinoma of the stomach which is a subtype of gastric tumors with hepatoid features and frequently producing $\alpha$-fetoprotein (AFP) [6, 7]. Herein, we report an interesting case of double malignancy, synchronous primary gastric cancer, and primary HCC with no evidence of pre-existing liver disease. To the best of our knowledge, there were only 2 cases in the literature describing this association $[8,9]$.

\section{Case Report}

A 50-year-old male patient presented to our department with a 6-month history of increasing epigastric pain with no radiation, vomiting, and unexplained weight loss of $6 \mathrm{~kg}$. His past medical, surgical, and familial histories were unremarkable, especially for malignancy. He had no habits of alcohol intake or tobacco consumption. He had a good socioeconomic status.

At presentation, the patient had a good general condition and his BMI was within the standard range $\left(B M I=22.8 \mathrm{~kg} / \mathrm{m}^{2}\right)$. He was hemodynamically stable with blood pressure of $130 / 70 \mathrm{~mm} \mathrm{Hg}$, pulse rate of 74 beats per minute, and body temperature of $37.5^{\circ} \mathrm{C}$.

On physical examination, the abdomen was soft with localized tenderness at the right upper quadrant. No abdominal mass or palpable pathologic lymph nodes were noted. There were no signs of portal hypertension or ascites. His routine laboratory tests on admission did not show any abnormal findings. His liver function tests and serum albumin (42 g/L) were within normal limits.

Upper gastrointestinal endoscopy was performed and revealed an ulcero-infiltrative growth in the antrum occluding the pylorus. There were no esophageal or gastric varices. Histopathologic examination showed poorly differentiated adenocarcinoma. There were no Helicobacter pylori or intestinal metaplasia.

For staging purposes, an abdominal CT scan was performed and showed irregular wall thickening of the antral wall and the pyloric channel associated with lymph nodes along the lesser curvature of the stomach (Fig. 1). There was a hypervascular mass lesion in the hepatic segment VIII $(50 \times 30 \mathrm{~mm})$ with enhancement during the arterial phase and washout during the venous phase, adjacent to the hepatic veins and the inferior vena cava, suggesting the diagnosis of HCC with no imaging features of liver cirrhosis. CT scan of the chest showed normal lungs, heart, and pleural spaces, and no evidence of metastatic lesions or lymphadenopathy.

Serological tests revealed absence of hepatitis B and C antigens. AFP and carcinoembryonic antigen levels were normal. In order to better characterize the hepatic mass, an MRI was performed and showed a typical enhancement pattern of HCC (Fig. 2). 
In the absence of clinical, biological, or imaging signs of cirrhosis, an ultrasonographyguided fine needle biopsy of the liver mass was performed and revealed pathological features in favor of HCC grade III according to the Edmondson-Steiner criteria. Histology of nontumor tissue showed absence of liver fibrosis. There were no eosinophilic or wide lamellar bands of fibrous tissue that are characteristic of the fibrolamellar HCC (Fig. 3).

The final diagnosis was made as double primary malignancies, consisting of gastric cancer and HCC. A multidisciplinary consultation was held. A total gastrectomy with eosojejunal anastomosis was decided without neoadjuvant chemotherapy as the tumor was complicated with pyloric stenosis.

The histopathological examination of the surgical specimen revealed a poorly differentiated adenocarcinoma with presence of signet-ring cell, infiltrating the entire gastric wall and the perigastric fat (Fig. 4). Eight lesser curvature lymph nodes were involved by metastatic carcinoma. The resection was judged complete, and the final tumor staging was pT3 N3a $(8 / 16) \mathrm{M} 0$. The overlying gastric mucosa was histologically unremarkable and resection margins were free of tumor. The postoperative course was uneventful and the patient was discharged after 1 week.

The HCC was considered unresectable because it invaded the vascular structures, particularly the vena cava. Therefore, the patient underwent transarterial chemoembolization (TACE) twice for HCC, with partial response ( $40 \%$ of necrosis) (Fig. 5). Thereafter, radiofrequency ablation was made for the residual lesion during outpatient follow-up. The patient is regularly followed up and was still in good general condition 2 years after the primary diagnosis.

\section{Discussion}

Synchronous malignancies including different organs are relatively rare compared to metastatic lesions. The incidence of gastric cancer with a synchronous second primary cancer varies from 2.0 to $10.9 \%$ [10].

HCC represents about $90 \%$ of all primary liver cancers. It develops on cirrhotic liver in $80 \%$ of cases and appears in $20 \%$ of patients with noncirrhotic chronic liver disease [11]. HCC can also develop in patients without evidence of chronic liver disease. This condition remains a relatively rare diagnosis [12]. This is consistent with the current case as our patient showed no evidence of pre-existing liver disease.

Synchronous occurrence of primary gastric cancer and HCC is a very rare condition. The underlying pathophysiology of multiple primary malignancies is still unknown. However, there are suggested hypothesis such as common carcinogens, genetic susceptibility, and family history of malignancy [13].

Diagnosis of synchronous primary gastric cancer with synchronous primary HCC is difficult without tissue diagnosis. The main differential diagnosis that must be considered is hepatoid adenocarcinoma of the stomach with a reported incidence of $1.3-15 \%$ of all gastric carcinomas [14]. It is a subtype of gastric tumors, with an extremely poor prognosis, characterized by hepatoid features and the production of large amounts of AFP [15]. In the case of our patient, we have discussed both the diagnosis of gastric hepatoid adenocarcinoma with liver metastasis and synchronous primary gastric cancer and primary HCC. However, taking into account the normal level of AFP and the absence of hepatoid features of the gastric tumor, we considered the second diagnosis. 
In summary, we have described an unusual case of synchronous gastric adenocarcinoma and HCC in noncirrhotic liver. It is obligatory to distinguish synchronous primary lesions from metastatic lesions for therapeutic purposes. Our patient underwent surgical treatment for his gastric cancer and was treated with both radiologic and medical interventions for his HCC.

\section{Statement of Ethics}

Informed consent was obtained from the participant involved in the study.

\section{Disclosure Statement}

The authors report no relevant conflicts of interest.

\section{Funding Sources}

This study was funded by the University Hospital of Sahloul (Sousse, Tunisia).

\section{Author Contributions}

Aya Hammami wrote the manuscript. Nour Elleuch, Aida Ben Slama, Hanen Jaziri, and Ahlem Braham were involved in drafting the manuscript or revising it critically for important intellectual content. Mehdi Ksiaa, Ali Jmaa, and Salem Ajmi gave final approval of the version to be published. Yasser Ben Cheikh provided and interpreted the radiological images. All authors certify that they qualify for authorship because of their substantial contribution to the work submitted. The authors declare that this paper has not been published nor is it under simultaneous consideration for publication elsewhere. The final version of the manuscript has been seen and approved by all authors.

\section{References}

1 Parkin DM. International variation. Oncogene. 2004 Aug;23(38):6329-40.

2 Ferlay J, Shin HR, Bray F, Forman D, Mathers C, Parkin DM. Estimates of worldwide burden of cancer in 2008: GLOBOCAN 2008. Int J Cancer. 2010 Dec;127(12):2893-917.

3 McLoughlin JM. Adenocarcinoma of the stomach: a review. Proc Bayl Univ Med Cent. 2004 Oct;17(4):391-9.

4 Balogh J, Victor D 3rd, Asham EH, Burroughs SG, Boktour M, Saharia A, et al. Hepatocellular carcinoma: a review. J Hepatocell Carcinoma. 2016 Oct;3:41-53.

5 Kabbage L, El Kouhen M, Taghy A, Znati K, Kabbaj N. A rare presentation of hepatocellular carcinoma in noncirrhotic liver. Pan Afr Med J. 2017 Sep;28:69.

6 Su JS, Chen YT, Wang RC, Wu CY, Lee SW, Lee TY. Clinicopathological characteristics in the differential diagnosis of hepatoid adenocarcinoma: a literature review. World J Gastroenterol. 2013 Jan;19(3):321-7.

7 Ahn JS, Jeon JR, Yoo HS, Park TK, Park CK, Sinn DH, et al. Hepatoid adenocarcinoma of the stomach: an unusual case of elevated alpha-fetoprotein with prior treatment for hepatocellular carcinoma. Clin Mol Hepatol. 2013 Jun;19(2):173-8.

8 Pandiaraja J. A case of synchronous primary gastric cancer and primary hepatocellular carcinoma. Indian J Med Paediatr Oncol. 2018 Jul;39(3):405-7.

9 Hu Z-R, Huang C-T, Chen W-Y, Wu H-C, Kuo Y-H, Li C-F, et al. Synchronous gastric cancer and hepatocellular carcinoma. J Cancer Res Pract. 2014 Dec;1(3):226-32. 
Hammami et al.: Synchronous HCC in a Patient with Primary Gastric Cancer

10 Lee JH, Bae JS, Ryu KW, Lee JS, Park SR, Kim CG, et al. Gastric cancer patients at high-risk of having synchronous cancer. World J Gastroenterol. 2006 Apr;12(16):2588-92.

11 European Association For The Study Of The Liver, European Organisation For Research and Treatment Of Cancer. EASL-EORTC clinical practice guidelines: management of hepatocellular carcinoma. J Hepatol. 2012 Apr;56(4):908-43.

12 Casanova-Martínez L, Castillo-Grau P, Jaquotot-Herranz M, Ruiz-Fernández G, Han EJ, Tortajada-Laureiro L, et al. Hepatocellular carcinoma in non-cirrhotic liver. Rev Esp Enferm Dig. 2012 Sep;104(9):505-6.

13 Chong VH, Idros A, Telisinghe PU. Triple synchronous gastrointestinal malignancies: a rare occurrence. Singapore Med J. 2010 Oct;51(10):e176-7.

14 Mehlman DJ, Bulkley BH, Wiernik PH. Serum alpha-1-fetoglobulin with gastric and prostatic carcinomas. N Engl J Med. 1971 Nov;285:1060-1.

15 Ishikura H, Fukasawa Y, Ogasawara K, Natori T, Tsukada Y, Aizawa M. An AFP-producing gastric carcinoma with features of hepatic differentiation. A case report. Cancer. 1985 Aug;56(4):840-8.

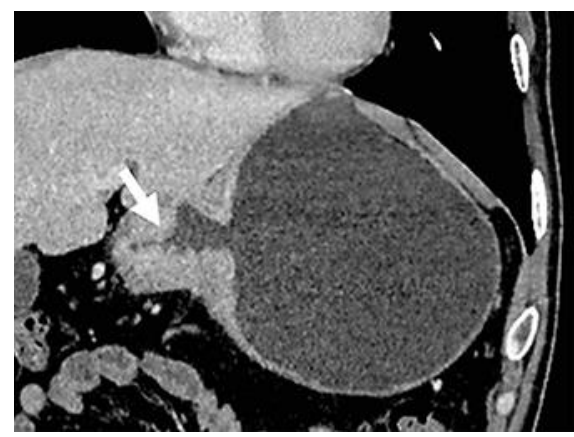

Fig. 1. CT findings showing irregular circumferential thickening of the antral wall (white arrow) and the pyloric channel.
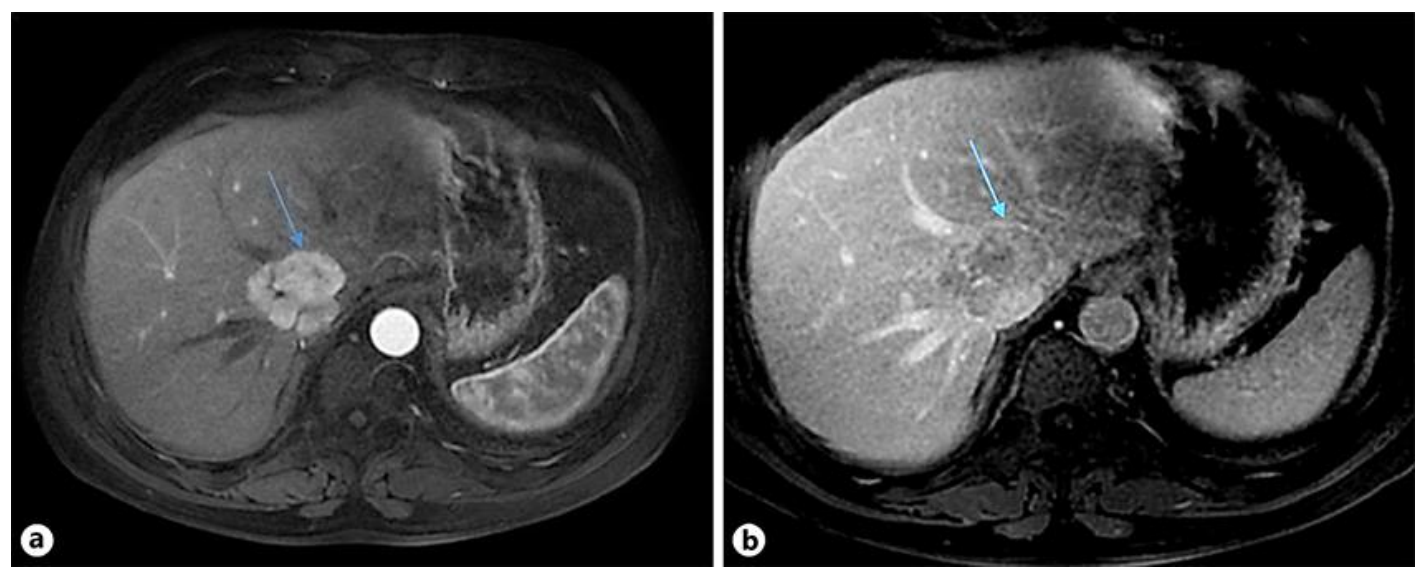

Fig. 2. T1-weighted 3D GRE images after gadolinium injection, showing a lesion in segment VIII with restriction of diffusion, intense enhancement on the arterial phase (a, blue arrow), and washout on the delayed phase (b, blue arrow) with an enhancing capsule. 


\section{Case Reports in Gastroenterology}

\begin{tabular}{l|l}
\hline Case Rep Gastroenterol 2020;14:299-305 \\
\hline DOI: 10.1159/000506188 & $\begin{array}{l}\text { @ 2020 The Author(s). Published by S. Karger AG, Basel } \\
\text { www.karger.com/crg }\end{array}$ \\
\hline
\end{tabular}

Hammami et al.: Synchronous HCC in a Patient with Primary Gastric Cancer
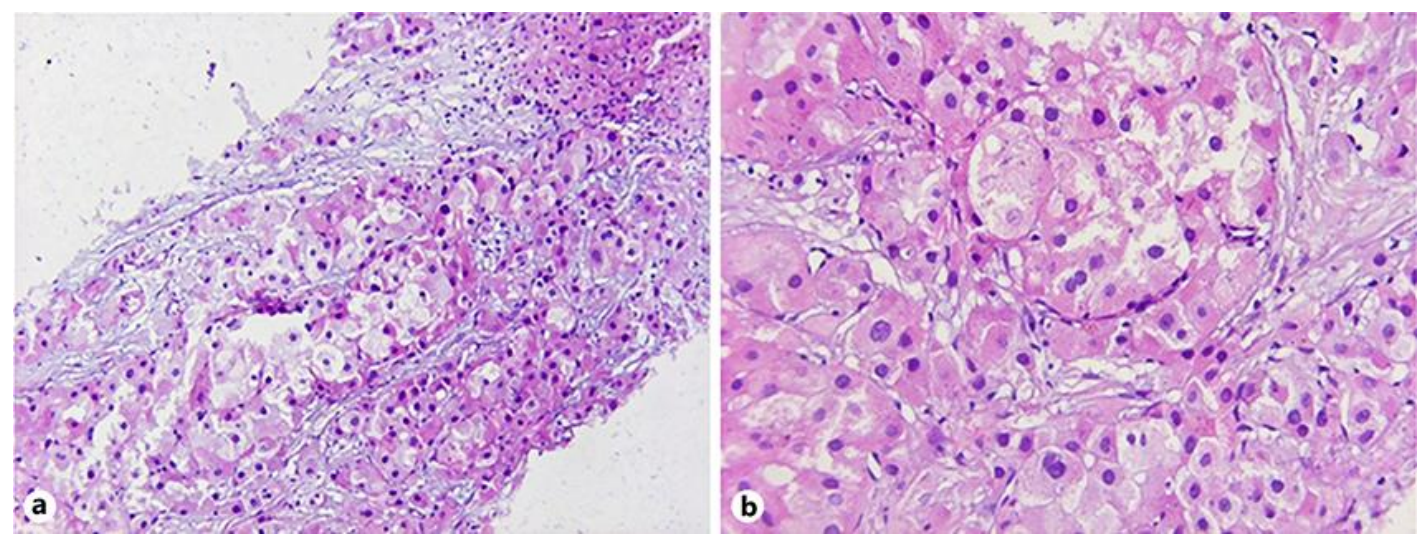

Fig. 3. a Proliferation of poorly organized hepatocytes dissociated by a fibrous stroma. HE. $\times 100$. b Hepatocytes with moderate nuclear atypia. HE. $\times 200$.
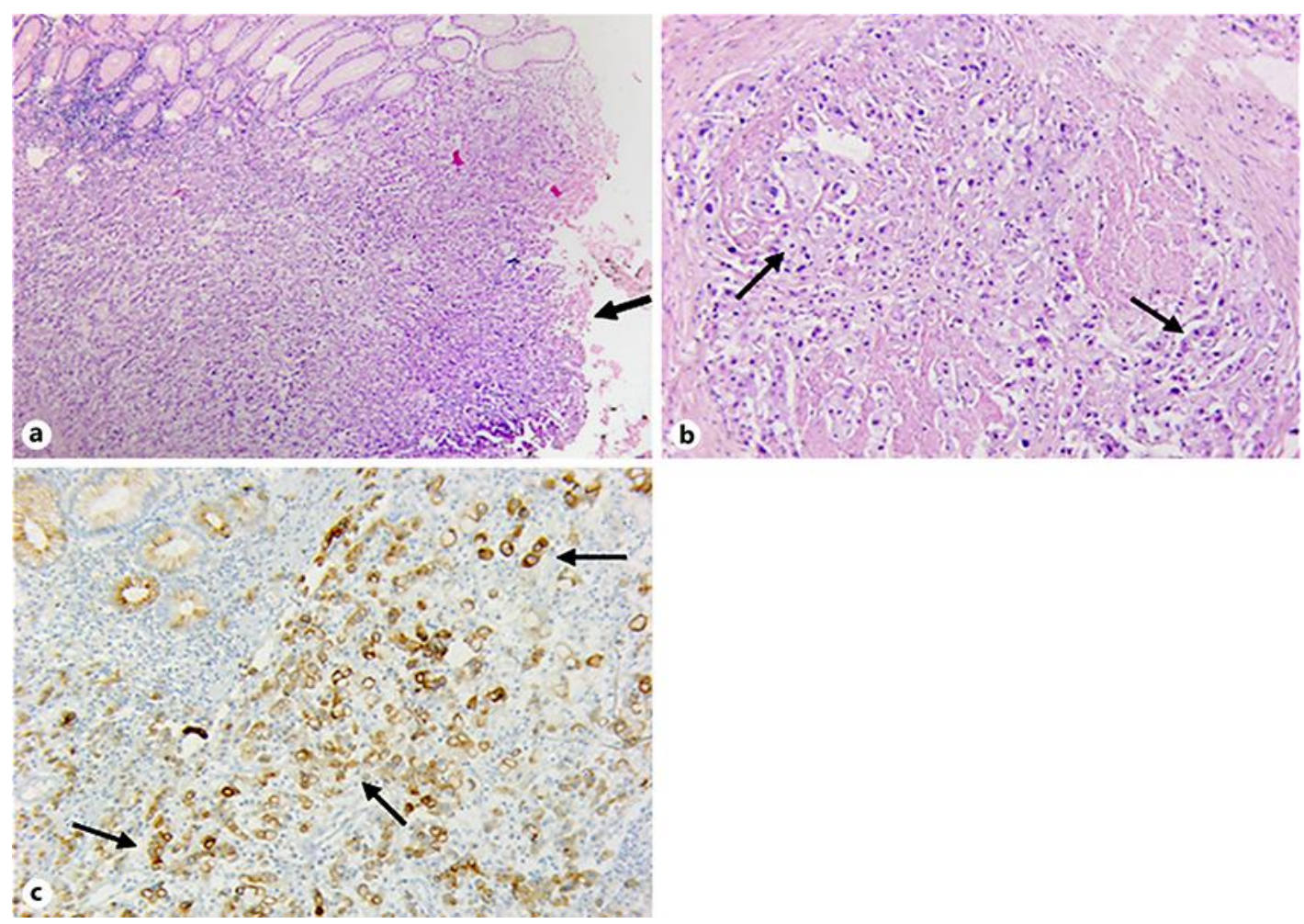

Fig. 4. a Ulceration of the gastric mucosa. HE. $\times 40$. $\mathbf{b}$ Infiltration of the muscle layers by tumor cells. HE. $\times 200$. c Diffuse cytoplasmic labeling of tumor cells. CK. $\times 100$. 

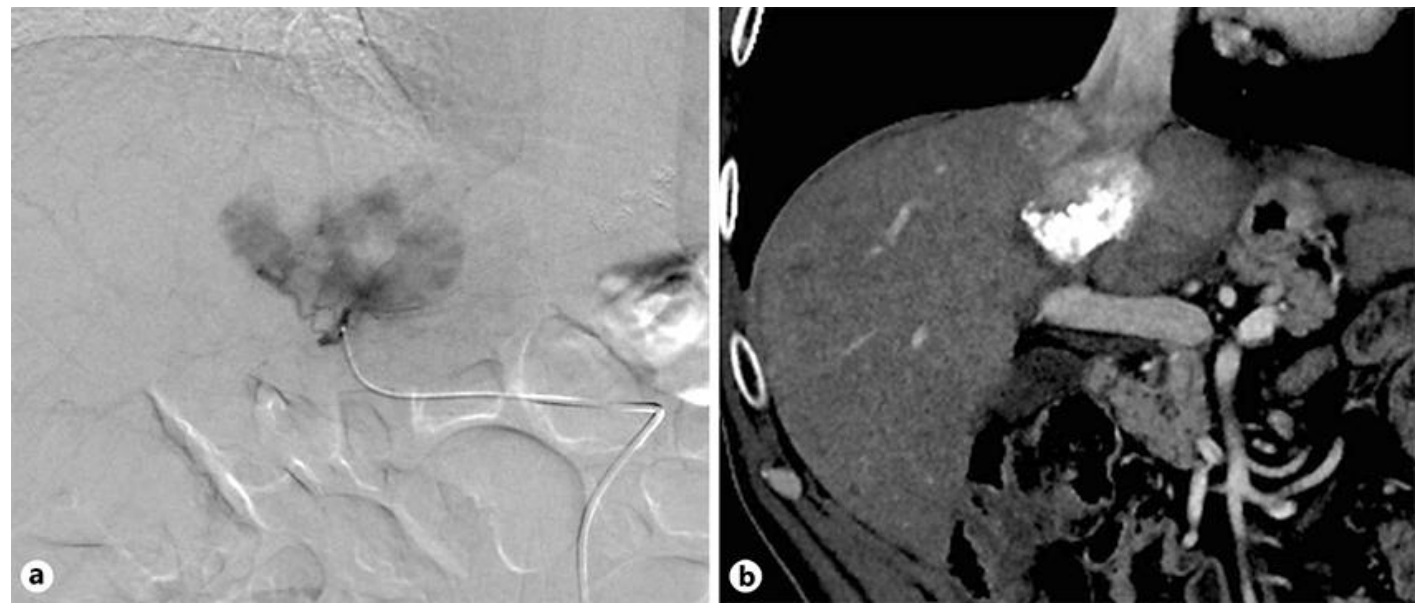

Fig. 5. a Superselective catheterization of the hepatic arterial branch feeding the tumor. Doxorubicin-in-oil emulsion was administered, followed by gelatin sponge particles. b CT images obtained 1 month after the procedure showing persistent active disease with moderate lipiodol retention within the target tumor. 